\title{
Well-being and perspective of second year MBBS students on online pharmacology classes held during COVID-19 pandemic in a tertiary care teaching hospital
}

\author{
Latha Kamath*, Nishith R. S., Ananya Chakraborty
}

Department of Pharmacology, Vydehi Institute of Medical Sciences and Research Centre, Bangalore, Karnataka, India

Received: 05 September 2021

Accepted: 29 September 2021

\author{
*Correspondence: \\ Dr. Latha Kamath, \\ Email: kamath.latha1@gmail.com
}

Copyright: ( $)$ the author(s), publisher and licensee Medip Academy. This is an open-access article distributed under the terms of the Creative Commons Attribution Non-Commercial License, which permits unrestricted non-commercial use, distribution, and reproduction in any medium, provided the original work is properly cited.

\begin{abstract}
Background: COVID-19 pandemic shifted all the classroom teaching to virtual online platforms. The overnight change in the teaching structure posed serious challenges especially for medical education. This study aims to assess the wellbeing of medical students undergoing online medical education during COVID-19 pandemic and their perspective on online pharmacology classes.

Methods: World health organization (WHO-5) well-being index was used to assess well-being of students. An internally validated questionnaire was used to assess student's perspective on online pharmacology classes. The questionnaire was administered to eligible consenting students online through Google forms. The data obtained was analysed by SPSS software.

Results: The mean wellness score $(\%)$ for all participants, $(n=118)$ was $48.87 \%$. The mean wellness score for males $(58.67 \%)$ was higher than for females $(42.41 \%)$. The average score for overall benefit of conducting online pharmacology classes was 3.32 out of 5. Objectively assessed online interactions like formative assessment, polls and quiz were rated higher than subjective interactions like debate.

Conclusions: COVID-19 pandemic has caused massive disruption in the life of many people. In our study, we report a decreased well-being score in medical students attending virtual classes. The findings on well-being of students have implications on planning redressal mechanism in such extreme situations. Our analysis of student's perspective about online interactions has implications beyond online classes. Some of the interactions can be instituted into regular curriculum increasing the student's participation.
\end{abstract}

Keywords: Pharmacology online classes, COVID-19 medical education, WHO well-being scale

\section{INTRODUCTION}

The novel severe acute respiratory syndrome coronavirus 2 (SARS-CoV-2) emerged in late 2019 and caused a disease called 'coronavirus disease 2019' (COVID-19). Being highly transmissible, COVID-19 spread at a very fast pace and brought the whole world to a standstill in $2020 .{ }^{1}$ On $11^{\text {th }}$ March 2020 the world health organization (WHO) declared COVID-19 as a pandemic, due to the rapid increase in the number of infected people with it. ${ }^{2}$ As of August 2021, it has affected nearly 213 million people and has claimed over 4 million lives worldwide. ${ }^{3}$

Most of the countries in the world imposed several restrictions to reduce the transmission of COVID-19; lockdowns have been announced in around 80 countries. $^{4}$ The restriction in movements affected the students across all boards. Medical education was particularly affected as the healthcare professional's activity was prioritised towards patient care in many of the institutions. ${ }^{5}$ As the world began to move towards more virtual meetings, the 
medical colleges everywhere began implementing virtual classes. Medical education that relies more on bedside learning was severely affected. ${ }^{6}$ The immense restriction in movements, coupled with uncertainties affected the psychological well-being of medical students. ${ }^{7}$

Most of the medical colleges in India started implementing online classes. The transition for preclinical and paraclinical curriculum was less daunting when compared to clinical curriculum. Nevertheless, few colleges struggled in all formats due to lack of infrastructure and redirection of staff towards COVID management. ${ }^{8}$ Apart from academics, some students were regularly involved in research projects. Many of such projects were either delayed or shelfed.$^{9}$

The implementation of online virtual classes was challenging in many colleges due to various issues. Factors like time constraints, poor technical skills, inadequate infrastructure and absence of institutional strategies impeded the implementation. ${ }^{10}$ Despite various hurdles, some colleges successfully implemented in the online transition. In one of the published studies, majority of the students preferred hybrid mode of teaching for post pandemic curriculum. ${ }^{11}$ There may be few learnings from online classes that can be extended into the normal curriculum.

In our Vydehi Institute of Medical Sciences and Research Centre, Bengaluru, we were quick to transition to online teaching schedule. Department of pharmacology undertook several innovative measures to improve the interaction and involvement of students during this period. We implemented several measures like formative assessments, quiz competitions, zoom polls, student chosen revision topics, student presentations among others.

This study was planned to evaluate wellness of students who have undergone extensive online classes through their current academic year. We also planned to evaluate the student's perspective on online classes in general and about the specific initiatives taken in department of pharmacology. The student's perspective will help in future planning of teaching models.

\section{METHODS}

A prospective, cross-sectional, observational, questionnaire-based study was carried out at Vydehi institute of medical sciences and research centre, Bangalore for a period of 1 month from $1^{\text {st }}$ February 2021 to $28^{\text {th }}$ February 2021. Sample size was calculated to be 97 using estimation technique. All $2^{\text {nd }}$ year MBBS students who were consenting to take part in the study were included. Students not willing to participate and $1^{\text {st }}, 3^{\text {rd }}$ and final year MBBS students, interns, postgraduate students and other healthcare professionals were excluded from our study.
A pilot of the questionnaires that were used in the study was completed before the study commenced. We piloted the questionnaires to ensure that they were acceptable and comprehensible to participants, and that our methods of administration were feasible and reliable.

After obtaining clearance from the institutional ethics committee, an online questionnaire was provided to all the $2^{\text {nd }}$ year MBBS students who were willing to participate. Google forms were used to administer questionnaire. WHO-5 well-being questionnaire was used to assess wellbeing. ${ }^{12,13}$ An internally validated questionnaire was used to assess student's perspective on online pharmacology classes.

\section{Study tools}

\section{The WHO-5, well-being index}

It is a short, self-administered questionnaire covering five positively worded items, related to positive mood (good spirits, relaxation), vitality (being active and waking up fresh and rested), and general interests (being interested in things). It has shown to be a reliable measure of emotional functioning. Administering the WHO-5 well-being index takes a few minutes. ${ }^{12}$

The raw score is calculated by summating the scores from the five answers and they have a range between 0 and 25 ( 0 : worst possible; 25 : best possible quality of life). To obtain a percentage score ranging from 0 to 100 , the raw score is multiplied by four. A percentage score of 0 represents the worst possible, whereas a score of 100 represents the best possible quality of life. A score of $\leq 50 \%$ indicates poor well-being and may require further assessment. $^{13}$

\section{Internally validated questionnaire}

A structured questionnaire was developed and used to collect data from the undergraduate medical students, which was made available electronically through Google forms.

There was a total of 23 questions in the questionnaire. It had a total of 6 main sections, which had questions related to socio-demographic details, online learning experience for pharmacology, technological difficulties, COVID-19 and its effects on learning, future prospects of online learning and COVID-19 and other activities. The students were asked to rate different aspects on a 5-point scale; 5 being the highest and 1 being the lowest.'

\section{Statistical analysis}

Data on student's response were subjected to statistical analysis. Statistical analysis was done using SPSS software Version 20. Descriptive Student's t-test like mean and median was used for continuous variables; frequency and percentage for categorical variables. Students $t$ test 
was used to compare the mean wellness score between male and females.

\section{RESULTS}

The total number of students who responded to questionnaire was 118 .

\section{Demographic details}

Of the total respondents, we had 88 (74.6\%) females and $30(25.4 \%)$ males. The mean age of participants was $20.44 \pm 0.88$ years. The participants were second year medical students.

\section{Mean wellness score}

The mean wellness score (percentage) for all participants was $48.87 \%$. The mean wellness score for males $(58.67 \%)$ was higher than that for females $(42.41 \%)$. The result was found to be statistically significant. The results are shown in Table 1.

Table 1: Demographic data and mean wellness score, $(\mathbf{n}=118)$.

\begin{tabular}{|llll|}
\hline Variables & $\begin{array}{l}\text { All, } \\
\text { n(\%) }\end{array}$ & $\begin{array}{l}\text { Female, } \\
\text { n(\%) }\end{array}$ & $\begin{array}{l}\text { Male, } \\
\text { n }(\%)\end{array}$ \\
\hline $\begin{array}{l}\text { Number of } \\
\text { participants }\end{array}$ & $\begin{array}{l}118 \\
(100)\end{array}$ & $88(74.6)$ & $30(25.4)$ \\
\hline $\begin{array}{l}\text { Mean age in } \\
\text { years (SD) }\end{array}$ & $\begin{array}{l}20.44 \\
(0.88)\end{array}$ & $\begin{array}{l}20.34 \\
(0.76)\end{array}$ & $\begin{array}{l}20.73 \\
(1.14)\end{array}$ \\
\hline $\begin{array}{l}\text { Mean wellness } \\
\text { score }\end{array}$ & 48.87 & $42.41^{*}$ & $58.67^{*}$ \\
\hline $\begin{array}{l}\text { Poor well- } \\
\text { being }\end{array}$ & $66(55.9)$ & $57(64.8)$ & $9(30)$ \\
\hline
\end{tabular}

$* p<0.05$

\section{Overall feedback for online pharmacology classes}

The average score for overall benefit of conducting online pharmacology classes was 3.32 out of 5 . The average score when asked individually for benefits of conducting theory and practical classes were 3.35 and 3.17 respectively. More students (45.8\% vs 12\%) agreed that online pharmacology classes imparted a right amount of theoretical knowledge when compared to practical knowledge.

The students rated an average score of 3.82 when asked about the overall interaction during online pharmacology classes and an average score of 3.60 for overall satisfaction for the online classes' format.

\section{Feedback on various unique interactive online classes}

The average rating of different online teaching methodologies adopted are shown in Figure 1. Objectively assessed interactions like formative assessment, polls and quiz were rated higher than subjective interactions like debate. (Figure 1).

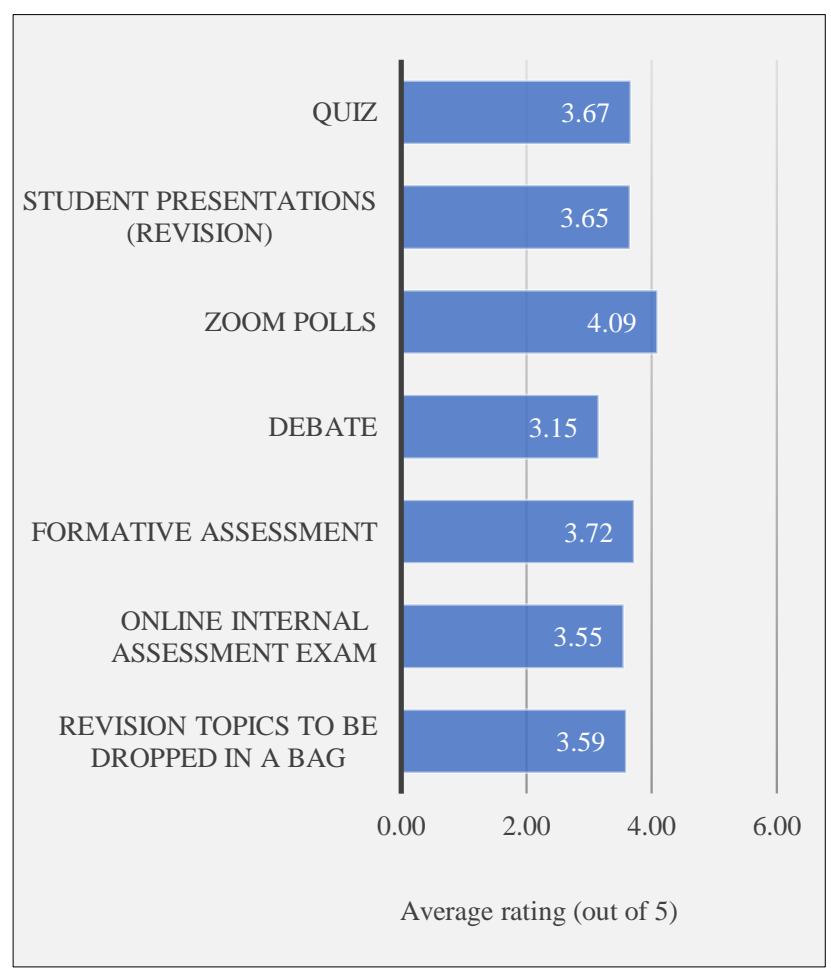

Figure 1: Average rating (out of 5) for different interactive teaching methodologies.

\section{Online classes: What it holds for offline classes}

Majority of the students (55.1\%) preferred a combination of offline classes and online assignments/ assessments going further.

Quiz (49.2\%), polls (54.2\%) and formative assessments $(45.8 \%)$ have been preferred by more students than other mode of interaction to be continued in the curriculum during offline classes.

\section{Online classes and COVID: The impact on health and other factors}

Majority of the students (72\%) spent an average of 3-5 hours/ day on online classes (Figure 2).

Only 7 students $(5.9 \%)$ had no health issues while remaining students complained of various issues as depicted in the (Figure 3).

Around $6(5.1 \%)$ students had a diagnosis of COVID whereas around 38 students $(32.2 \%)$ had one or more of the immediate family members getting a diagnosis of COVID infection.

Around $70 \%$ of students involved in research activities have stated that COVID has adversely impacted their research progress. Around $72.9 \%$ of students involved in 
sports/extracurricular activities have stated that COVID has adversely impacted their activities.

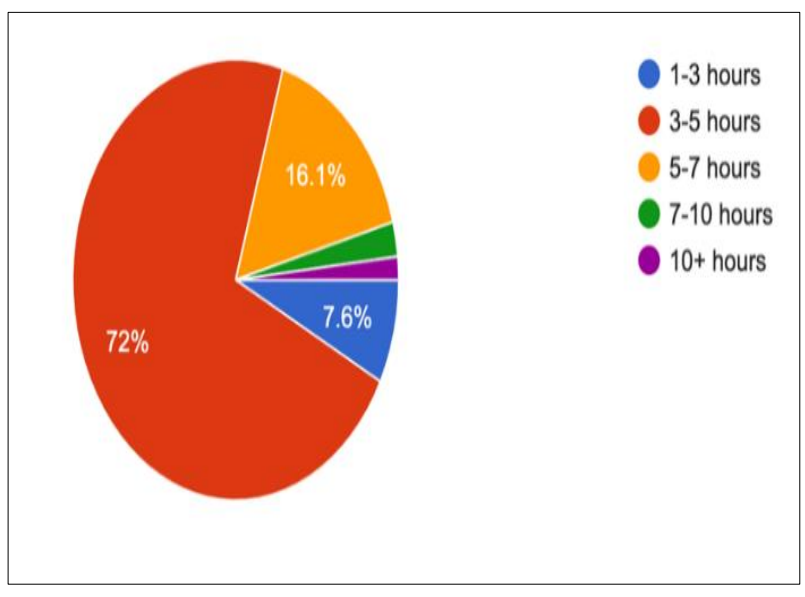

Figure 2: Average number of hours students spend on online classes/day.

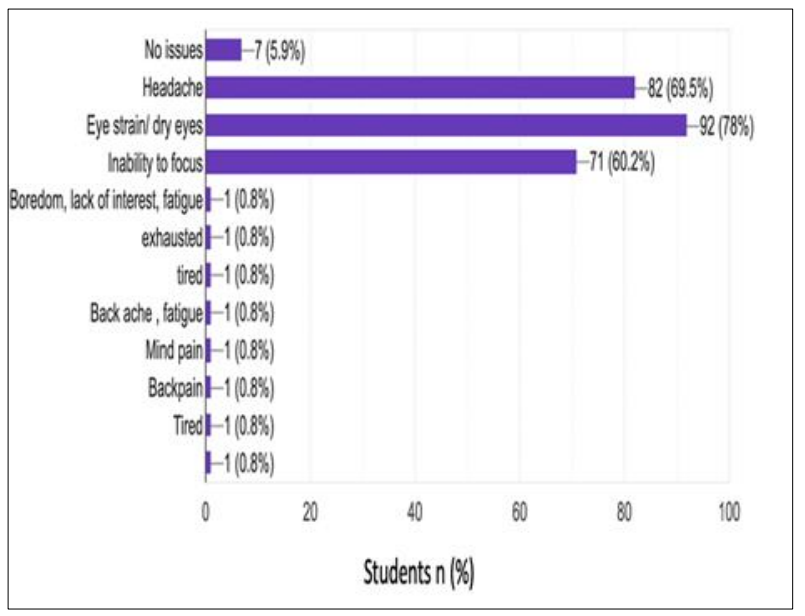

Figure 3: Different health issues reported by students after attending online classes followed by, $n(\%)$.

\section{DISCUSSION}

In our study, we investigated the well-being of medical students during COVID pandemic who have been undergoing online classes. The mean wellness score for all students was around $48.87 \%$. The mean wellness score of medical students in a separate study conducted by Mirza et al was around $70 \%$ in pre COVID era. ${ }^{14}$ The lower overall mean wellness score observed in our study points towards the significant impact of the COVID pandemic on the wellbeing of the medical students. Similarly, psychological domain was affected the most in one of the studies conducted to access the quality of life of medical students during COVID pandemic in India using WHO-QOL BREF questionnaire. ${ }^{7}$ In our study, around $66(55.9 \%)$ students had a mean wellness score of $<50 \%$ indicating poor well-being. That translates to more than half of the students requiring further evaluation for their poor well- being indicating a heightened burden of COVID pandemic on student's well-being.

We noted a significant difference between gender with respect to number of people reporting mean wellness score below 50\%. About 57 (64.8\%) of females reported mean wellness score below $50 \%$ when compared to only $9(30 \%)$ of males. These findings are different from that of study done by Mirza et al in pre COVID era. Mirza et al reported the mean wellness score for females $(71.8 \%)$ was slightly higher than for males $(68.8 \%)$. In a study done in pre COVID era in a medical college in India, the perceived stress was higher among females when compared to males. ${ }^{15}$ The COVID pandemic related stress may be affecting female medical students more than males. Also, we had more of female participants in the study compared to males.

In our study, nearly $78 \%$ of the students complained of eye strain/ dry eyes. In a study conducted by Premchander et al on all medical students of our institution before COVID pandemic, the prevalence of dry eye disease (DED) was only around $39.1 \% .^{16}$ More screen time due to online virtual classes may be the contributing factor for increased dry eye related complaints. We are planning to conduct a session in association with department of ophthalmology to address the issue.

In a study conducted by Lawande et al on medical students of various medical colleges in India, around $46.3 \%$ of students felt that online medium is not suitable for practical classes. ${ }^{8}$ In our study also, around $49.6 \%$ of students felt that online classes were not able to impart right amount of practical knowledge. In this context, there is a need to further enhance the quality of practical classes. Setting up of virtual practical learning environment and laboratory simulations are expensive process. Though the virtual learning may never be able to replace the practical experience to medical students, there is a need to address this gap in these challenging times.

Study conducted by Hameed et al in a medical college in India concluded that online learning was not preferred by medical students and cited lack of interaction as the most likely reason. ${ }^{11} \mathrm{We}$ realized the need to engage students interactively and adopted various modes to interact with students virtually. Objectively assessed interactions like formative assessment, polls and quiz were rated higher than subjective interactions like debate by the students (Figure 1).

In our study, around $55.1 \%$ preferred a combination of offline classes and online assignments/ assessments going further. This was similar in the study done by Lawande et al. where around $60 \%$ of students had expressed interest in continuation of e-modules.

We also asked students which initiatives they would like to be continued in our normal curriculum. The students 
preferred quiz, polls and formative assessment to be continued.

Majority of the students (72\%) spent an average of 3-5 hours/day on online classes. We asked the students about health issues associated with long exposure to online classes. Only 7 students $(5.9 \%)$ had no health issues while remaining students complained of various issues as depicted in the Figure 2. The higher rate of physical issues associated with increased screen time may also would have contributed to lower well-being score in our study.

The COVID infection may have played a role in adversely impacting well-being of our students as $6(5.1 \%)$ students had a diagnosis of COVID whereas around 38 students $(32.2 \%)$ had one or more of the immediate family members getting a diagnosis of COVID infection.

COVID pandemic has affected various research-based projects undertaken by medical students. Our study also echoed similar result as $70 \%$ of students involved in research activities have stated that COVID has adversely impacted their research progress. The lack of mobility has also affected the sports/extracurricular activities that could have an impact on well-being. In our study, $72.9 \%$ of students involved in sports/extracurricular activities have stated that COVID has adversely impacted their activities.

\section{CONCLUSION}

Our study was unique in analyzing the student's perspective on different types of online interactions. These findings have implications beyond online classes. Some of the interactions can be instituted into regular curriculum increasing the student's participation.

The main weakness of our study was limited sample size as we restricted our study to only $2^{\text {nd }}$ year medical students and student's perspective assessment was limited to only pharmacology classes. Nevertheless, the findings on wellbeing of students have implications on planning redressal mechanism in such extreme situations.

\section{ACKNOWLEDGEMENTS}

Author would like to thank Principal, Vydehi Institute Of Medical Sciences \& Research Centre, Bangalore and RC for the permission to undertake the study. Also, thanks to members of ethics committee to agree to review our protocol on an expedited basis despite lockdown and our students who participated in the study.

Funding: No funding sources Conflict of interest: None declared

Ethical approval: The study was approved by the Institutional Ethics Committee

\section{REFERENCES}

1. Hu B, Guo H, Zhou P, Shi Z-L. Characteristics of SARS-cov-2 and COVID-19. Nat Rev Microbiol. 2021;19(3):141-54.

2. WHO. Coronavirus (COVID-19); Events as they Happen. Available from: https://www.who.int/emergencies/diseases/novelcoronavirus-2019/events-as-they-happen. Accessed on 2021 May 08.

3. WHO Coronavirus (COVID-19) Dashboard. Available at: https://covid19.who.int/. Accessed on 2021 August 27.

4. Han E, Tan MMJ, Turk E, Sridhar D, Leung GM, Shibuya K et al. Lessons learnt from easing COVID19 restrictions: an analysis of countries and regions in Asia Pacific and Europe. Lancet. 2020;396(10261):1525-34.

5. Sahi PK, Mishra D, Singh T. Medical Education Amid the COVID-19 Pandemic. Indian Pediatr. 2020;57(7):652-7.

6. Alsoufi A, Alsuyihili A, Msherghi A, Elhadi A, Atiyah H, Ashini A et al. Impact of the COVID-19 pandemic on medical education: Medical students' knowledge, attitudes, and practices regarding electronic learning. Plos One. 2020;15(11):1-20.

7. Chawla B, Chawla S, Singh H, Jain R, Arora I. Is coronavirus lockdown taking a toll on mental health of medical students? A study using WHOQOL-BREF questionnaire. J Fam Med Prim Care. 2020;9(10):5261-6.

8. Lawande NN, Kenkre TD, Mendes NA, Dias LM, Dias AS. Perspectives of Indian medical students on e-learning as a tool for medical education in the country: a quantitative study. Int J Community Med Public Heal. 2020;7(9):3610-16.

9. Rainbow S, Dorji T. Impact of COVID-19 on medical students in the United Kingdom. Germs. 2020;10(3):240-3.

10. Li HOY, Bailey AMJ. Medical Education Amid the COVID-19 Pandemic: New Perspectives for the Future. Acad Med. 2020;95(11):11-2.

11. Hameed T, Husain M, Kumar Jain S, Singh CB, Khan S. Maedica-a Journal of Clinical Medicine Online Medical Teaching in COVID-19 Era: Experience and Perception of Undergraduate Students. Maedica A J Clin Med. 2020;15(4):440-4.

12. Worobetz A, Retief PJ, Loughran S, Walsh J, Casey M, Hayes $\mathrm{P}$ et al. A feasibility study of an exercise intervention to educate and promote health and wellbeing among medical students: The "MED-WELL" programme. BMC Med Educ. 2020;20(1):1-12.

13. Omani-Samani R, Maroufizadeh S, Almasi-Hashiani A, Sepidarkish M, Amini P. The WHO-5 Well-Being Index: A Validation Study in People with Infertility. Iran J Public Health. 2019;48(11):2058-64.

14. Mirza W, Mirza AM, Saleem MS, Chacko PP, Ali M, Tarar MN et al. Well-being Assessment of Medical Professionals in Progressive Levels of Training: 
Derived from the WHO-5 Well-being Index. Cureus. 2018;10(12):1-9.

15. Anuradha R, Dutta R, Dinesh Raja J, Sivaprakasam P, Patil AB. Stress and stressors among medical undergraduate students: A cross-sectional study in a private medical college in Tamil Nadu. Indian $\mathrm{J}$ Community Med. 2017;42(4):222-5.

16. Premchander A, Chakraborty A, Naik V, Kolli H, Acharya S. Prevalence and risk factors of dry eye disease symptoms among medical undergraduates in south India. Int J scie res. 2020;9(2):16-8.
Cite this article as: Kamath L, Nishith RS, Chakraborty A. Well-being and perspective of second year MBBS students on online pharmacology classes held during COVID-19 pandemic in a tertiary care teaching hospital. Int J Basic Clin Pharmacol 2021;10:1276-81. 